\title{
openheart Dexrazoxane preferentially mitigates doxorubicin cardiotoxicity in female children with sarcoma
}

\author{
Hari K Narayan, ${ }^{\oplus 1,2}$ Mary E Putt, ${ }^{3}$ Nikitha Kosaraju, ${ }^{4}$ Alejandro Paz, ${ }^{4}$ Shivani Bhatt, ${ }^{5}$ \\ Theodore Plappert, ${ }^{6}$ Laura Mercer-Rosa, ${ }^{7}$ Saro H Armenian, ${ }^{8}$ Ami V Desai, ${ }^{9}$ \\ Richard B Womer, ${ }^{5,10}$ Bonnie Ky ${ }^{11}$
}

\begin{abstract}
- Additional material is published online only. To view these files, please visit the journal online (http://dx.doi. org/10.1136/openhrt-2019001025).
\end{abstract}

To cite: Narayan HK, Putt ME, Kosaraju N, et al. Dexrazoxane preferentially mitigates doxorubicin cardiotoxicity in female children with sarcoma. Open Heart 2019;6:e001025. doi:10.1136/ openhrt-2019-001025

Received 1 February 2019 Revised 27 March 2019 Accepted 26 April 2019

Check for updates

\section{C) Author(s) (or their} employer(s)) 2019. Re-use permitted under CC BY-NC. No commercial re-use. See rights and permissions. Published by BMJ.

For numbered affiliations see end of article.

\section{Correspondence to}

Bonnie Ky; Bonnie.Ky@uphs. upenn.edu

\section{ABSTRACT:}

Objective We sought to determine how sex and dexrazoxane therapy influence cardiac remodelling in children with sarcoma receiving high-dose doxorubicin. Methods In a retrospective cohort of 85 children with sarcoma receiving high-dose doxorubicin, echocardiography measures prior to, early after (within 6 months of doxorubicin completion) and $1-2$ years after doxorubicin completion were quantified. At each follow-up visit, multivariable, propensity-adjusted linear regression models evaluated dexrazoxane's effects on changes in left ventricular (LV) shortening fraction (SF), structure, strain and wall stress for subgroups divided by sex. Likelihood ratio tests assessed the interaction between sex and dexrazoxane in determining these changes.

Results Early after doxorubicin completion, males not treated with dexrazoxane $(n=15)$ developed increased cavity size and diminished circumferential strain; females ( $n=8$ ) developed diminished SF and strain indices, and increased cavity size and wall stress. With dexrazoxane, males $(n=33)$ demonstrated less deterioration in circumferential strain by $3.4 \%(95 \% \mathrm{Cl} 0.01$ to 6.8$)$, and females $(n=29)$ demonstrated less reduction in SF by $5.7 \%$ (95\% Cl 2.1 to 9.3), and had mitigation of increases in cavity size and wall stress. In interaction analyses, females had greater protection with dexrazoxane with regard to SF $(p=0.019)$ and cavity size in diastole $(p=$ $0.002)$ and systole $(p \leq 0.001)$. These findings largely persisted $1-2$ years after doxorubicin therapy. Conclusions Early, sustained alterations in LV structure and function occur in children with sarcoma after highdose doxorubicin, with adverse changes and protective effects of dexrazoxane more pronounced in females as compared with males. Dexrazoxane may have sex-specific cardioprotective effects.

\section{INTRODUCTION}

Anthracyclines are commonly used in the treatment of childhood cancers, but they confer a substantial risk of dose-dependent cardiotoxicity and associated cardiovascular morbidity and mortality. ${ }^{2}$ Children with sarcoma, a highly aggressive malignant solid tumour, are often treated with some of the highest cumulative anthracycline doses-on the order of

\section{Key questions}

What is already known about this subject?

- Anthracycline chemotherapy can cause dose-dependent cardiotoxicity and children with sarcoma are treated with some of the highest doses of these agents.

What does this study add?

- In this retrospective longitudinal cohort study, we performed detailed characterisation of echocardiography measures of cardiac structure and function before, early after and 1-2 years after high-dose anthracycline therapy in children with sarcoma. Female children were particularly susceptible to adverse changes in cardiac structure and function, and derived preferential benefit from dexrazoxane, a cardioprotectant given concurrently with anthracycline therapy. Early adverse changes in strain measures were associated with later declines in shortening fraction.

How might this impact on clinical practice?

- With further research, sex may be considered as a factor in the decision-making process of whether to use dexrazoxane. This study, in conjunction with published data in adults, provides support for the use of echocardiographic strain indices in the early monitoring of anthracycline-exposed children.

$375-450 \mathrm{mg} / \mathrm{m}^{2}$ of doxorubicin-and are thus particularly vulnerable to cardiac injury. ${ }^{34}$ Anthracycline-treated children may develop deterioration of left ventricular (LV) shortening fraction (SF), a surrogate of global LV systolic function, in addition to adverse changes in LV structure, contractility and afterload, each of which contribute to global systolic function. ${ }^{56}$

Dexrazoxane, an intracellular iron chelator, inhibits anthracycline-induced free-radical injury and topoisomerase II beta mediated DNA damage, and may be protective against these cardiotoxic effects. ${ }^{7-10}$ Female children may be particularly vulnerable to anthracycline cardiotoxicity, ${ }^{11}$ and may preferentially benefit from 


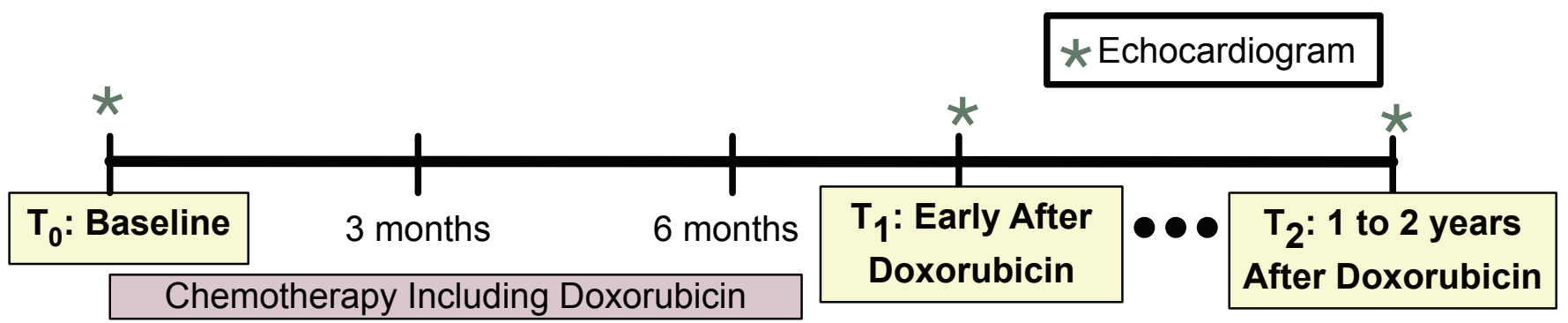

Figure 1 Timing of echocardiography acquisition in relation to chemotherapy. Approximate timing of quantitated echocardiograms in relation to doxorubicin exposure.

dexrazoxane. ${ }^{9}$ However, prior studies have focused primarily on lower anthracycline doses than sarcoma patients often receive, and important questions remain. The early and sustained effects of high-dose anthracyclines in paediatric sarcoma patients on measures of cardiovascular function and remodelling, including strain, and how these effects differ by sex and dexrazoxane therapy exposure, remain incompletely understood. A more robust understanding of these effects could help inform monitoring and treatment strategies.

Our objective was to characterise the effects of sex and dexrazoxane exposure in influencing early changes in $\mathrm{LV}$ function and remodelling after high-dose doxorubicin therapy for paediatric sarcoma. To accomplish this objective, we performed a retrospective, longitudinal cohort study examining detailed quantitative echocardiography assessments over standardised follow-up time intervals.

\section{MATERIALS AND METHODS Study population}

Via an institutional cancer database search, a retrospective cohort of sarcoma patients at the Children's Hospital of Philadelphia (Philadelphia, PA, USA) was identified who met the following eligibility criteria: (1) sarcoma diagnosis (Ewing sarcoma, osteosarcoma, rhabdomyosarcoma

\begin{tabular}{|c|c|c|c|}
\hline Characteristic & No Dexrazoxane* $(n=23)$ & Dexrazoxane $^{*}(n=62)$ & $\mathbf{P}^{\dagger}$ \\
\hline Age, years & $13.1(4.3)$ & $12.9(5.3)$ & 0.86 \\
\hline Female Sex & $8(35)$ & $29(47)$ & 0.32 \\
\hline \multicolumn{4}{|l|}{ Race } \\
\hline White & $17(74)$ & $47(76)$ & \multirow[t]{3}{*}{0.94} \\
\hline Black & $4(17)$ & $9(14)$ & \\
\hline Other & $2(9)$ & $6(10)$ & \\
\hline Prior anthracycline exposure & $0(0)$ & $1(2)$ & 0.54 \\
\hline \multicolumn{4}{|l|}{ Cancer Diagnosis } \\
\hline Ewing Sarcoma & $10(44)$ & $24(39)$ & \multirow[t]{4}{*}{0.42} \\
\hline Osteosarcoma & $9(39)$ & $21(34)$ & \\
\hline Rhabdomyosarcoma & $0(0)$ & $7(11)$ & \\
\hline Other soft tissue sarcoma & $4(17)$ & $10(16)$ & \\
\hline \multicolumn{4}{|l|}{ Cancer Stage } \\
\hline Localised & $15(65)$ & $48(77)$ & \multirow[t]{3}{*}{0.33} \\
\hline Metastatic to lungs & $5(22)$ & $6(10)$ & \\
\hline Widely metastatic & $3(13)$ & $8(13)$ & \\
\hline Cumulative Doxorubicin Dose, mg/m²(median (IQR)) & $375(375,450)$ & $375(375,450)$ & $0.24^{\ddagger}$ \\
\hline Chest radiation exposure & 2 (9) & $5(8)$ & 0.93 \\
\hline Body surface area, $\mathrm{m}^{2}$ & $1.55(0.46)$ & $1.46(0.50)$ & 0.50 \\
\hline Body mass index, $\mathrm{kg} / \mathrm{m}^{2}$ & $20.8(4.5)$ & $21.2(6.0)$ & 0.76 \\
\hline Systolic blood pressure, $\mathrm{mmHg}$ & $117(13)$ & $113(15)$ & 0.31 \\
\hline Diastolic blood pressure, mmHg & $63(10)$ & $64(10)$ & 0.62 \\
\hline
\end{tabular}

${ }^{*} P$-values from two-sided t-tests or Pearson's $\chi^{2}$ tests except where specified.

$\dagger P$-value from Wilcoxon rank sum test.

$\ddagger$ Mean (SD) or number of subjects (\% of total) except where specified.

IQR, IQR range. 


\begin{tabular}{|c|c|c|c|}
\hline Echocardiography Measure & No Dexrazoxane* $(n=23)$ & Dexrazoxane* $(n=62)$ & $\mathbf{P}^{\dagger}$ \\
\hline Shortening Fraction, $\%$ & $33.0(4.2)$ & $30.1(5.5)$ & 0.025 \\
\hline LVIDD, cm/BSA ${ }^{0.45}$ & $3.8(0.4)$ & $3.9(0.3)$ & 0.1 \\
\hline LVIDS, cm/BSA ${ }^{0.45}$ & $2.5(0.3)$ & $2.7(0.2)$ & 0.002 \\
\hline Posterior Wall Thickness, cm/BSA ${ }^{0.4}$ & $0.6(0.1)$ & $0.6(0.1)$ & 0.5 \\
\hline LV Mass, gr/BSA ${ }^{1.25}$ & $63(18)$ & $65(13)$ & 0.53 \\
\hline Relative Wall Thickness & $0.33(0.07)$ & $0.31(0.06)$ & 0.19 \\
\hline Circumferential Strain, \% & $-25.1(3.3)$ & $-23.0(4.1)$ & 0.045 \\
\hline Radial Strain, \% & $39.6(18.0)$ & $41.1(25.9)$ & 0.82 \\
\hline Meridional ESS, $10^{3}$ dynes $/ \mathrm{cm}^{2}$ & $83(17)$ & $98(30)$ & 0.031 \\
\hline Circumferential ESS, $10^{3}$ dynes $/ \mathrm{cm}^{2}$ & $136(20)$ & $149(27)$ & 0.064 \\
\hline
\end{tabular}

${ }^{*} P$-values from two-sided two-sample t-tests comparing no dexrazoxane to dexrazoxane.

†Mean (SD).

BSA, body surface area; ESS, end-systolic stress; LV, left ventricular; LVIDD, LV inner dimension in diastole; LVIDS, LV inner dimension in systole.

or non-rhabdomyosarcoma soft tissue sarcoma) made between 1 January 2006 and 1 February 2015; (2) treatment including at least $150 \mathrm{mg} / \mathrm{m}^{2}$ of doxorubicin and completely administered at the Children's Hospital of Philadelphia and (3) archived echocardiographic images at baseline before doxorubicin initiation and after completion. Clinical data through 1 February 2016 were extracted through a comprehensive chart review.

Subjects were treated with standard chemotherapy regimens as determined by the treating clinician. Regimens typically included a total of $375-450 \mathrm{mg} / \mathrm{m}^{2}$ of doxorubicin given over a period of approximately 6 months. Within each cycle, doxorubicin was administered either as (1) a $75 \mathrm{mg} / \mathrm{m}^{2}$ continuous infusion over 48 hours; or (2) two $37.5 \mathrm{mg} / \mathrm{m}^{2}$ doxorubicin boluses given 24 hours apart, each preceded by $375 \mathrm{mg} / \mathrm{m}^{2}$ of dexrazoxane (10:1 dexrazoxane:doxorubicin dosage ratio). Prior to August 2009, the majority of subjects were given continuous infusion doxorubicin without dexrazoxane; at the treating provider's discretion, some subjects received bolus doxorubicin with dexrazoxane during this time period. As per a change in institutional protocol, beginning in August 2009, all subjects were given bolus doxorubicin with dexrazoxane. Apart from dexrazoxane, there were no significant changes in treatment regimens over the study period. Given that $30 \%$ of subjects in the earlier era and $73 \%$ of the overall cohort received dexrazoxane, there was an opportunity to evaluate the effects of this drug. The local Institutional Review Board approved this study.

\section{Echocardiography}

Clinical echocardiograms were routinely performed to monitor for cardiotoxicity. Archived echocardiography images (30 frames per second, using a similar approach to prior studies ${ }^{13}$ from three time points were obtained: prior to doxorubicin initiation $\left(\mathrm{T}_{0}\right)$; early after (within 6 months of) doxorubicin completion $\left(T_{1}\right)$ and 1-2 years after doxorubicin completion
$\left(\mathrm{T}_{2}\right.$; figure 1). Echocardiograms were quantitated at the University of Pennsylvania Center for Quantitative Echocardiography (Philadelphia) by a single observer, blinded to subject characteristics. Quantitation details are provided in the online supplementary Methods.

Cavity size (LV inner dimension in diastole (LVIDD), LV inner dimension in systole (LVIDS)), myocardial wall size (posterior wall thickness and LV mass) and the ratio of wall thickness to cavity diameter (relative wall thickness) were quantitated to characterise structural remodelling. ${ }^{15}$ To provide further insight into systolic function, peak systolic strain was determined in the circumferential and radial dimensions from the parasternal short axis views. ${ }^{16}$ Wall stress, an index of afterload, was assessed via measures of meridional and circumferential end-systolic stress (ESS) ${ }^{17}$ Finally, SF, an assessment of global systolic function, was calculated from LVIDD and LVIDS. ${ }^{15}$ Longitudinal strain and ejection fraction were not analysed due to limitations in image quality in the apical views.

In order to account for normal growth, LVIDD, LVIDS, posterior wall thickness and LV mass were divided by body surface area (BSA) to the power of $0.45,0.45,0.40$ and 1.25 respectively, adjustments that were derived from a prior study of normal children. ${ }^{18}$ In this report, we characterise the BSA-adjusted values of these parameters.

\section{Statistical methods}

Standard descriptive statistics characterised the study population at baseline. Two sample t-tests or Wilcoxon rank sum tests (for continuous variables) and Pearson's $\chi^{2}$ tests (for categorical variables) were used to test for baseline differences by dexrazoxane exposure status. We either stratified by or adjusted for sex and dexrazoxane exposure in all analyses, anticipating differences between these groups based on previously published findings. ${ }^{9} 1112$ 


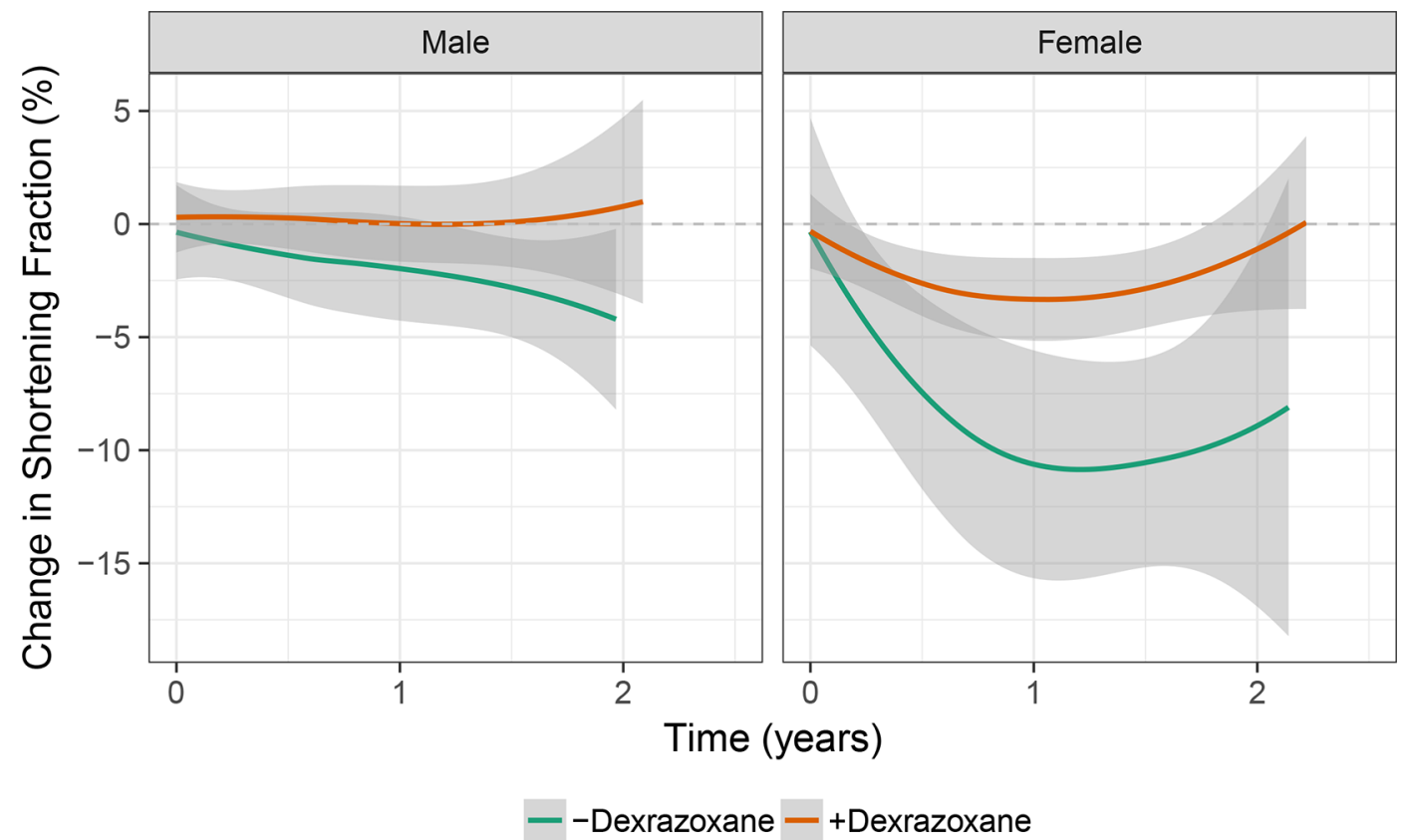

Figure 2 Changes in shortening fraction over time. in subgroups divided by sex and dexrazoxane exposure, LOESS smoothed curves with $95 \%$ Cls depict changes in shortening fraction over time.

A

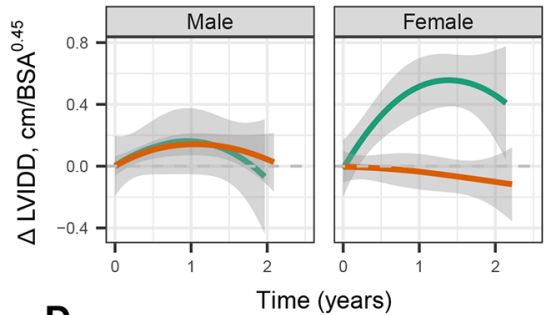

D

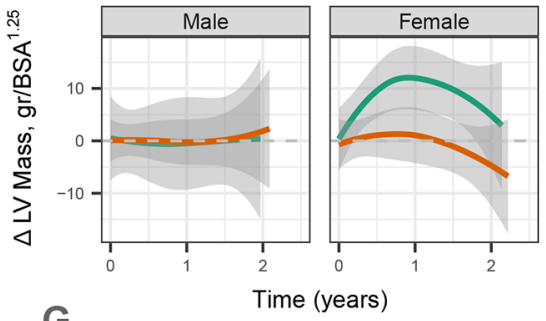

G

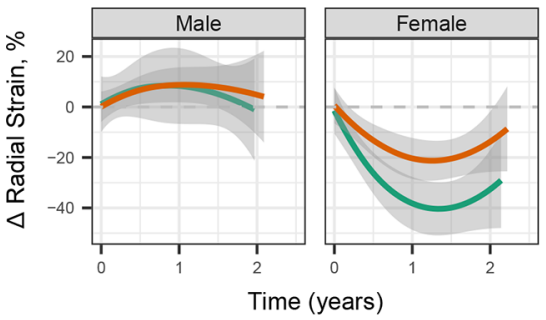

B

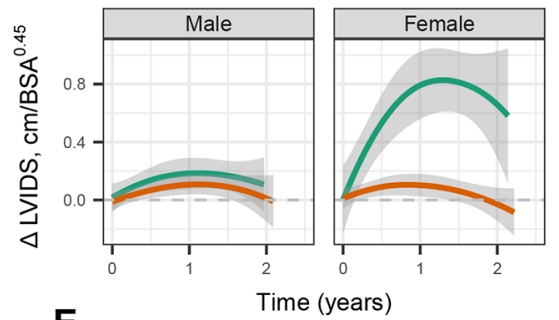

E

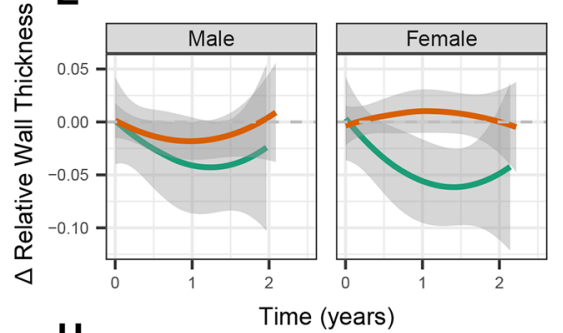

H

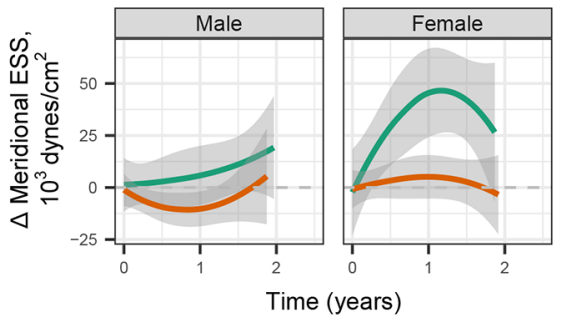

C

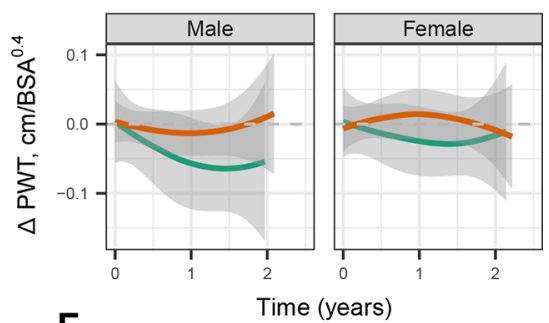

$\mathbf{F}$

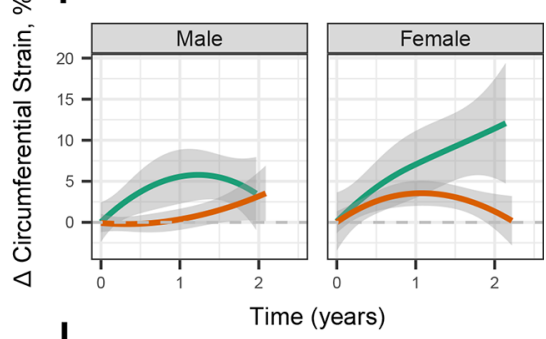

1

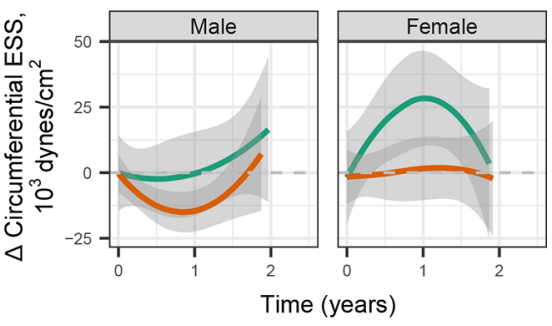

Time (years)

- -Dexrazoxane - +Dexrazoxane

Figure 3 Changes in structure, strain and wall stress over time. In subgroups divided by sex and dexrazoxane exposure, LOESS smoothed curves with 95\% Cls depict changes in A-left ventricular (LV) inner dimension in diastole (LVIDD); B-LV inner dimension in systole (LVIDS); C-posterior wall thickness (PWT); D-LV mass; E-relative wall thickness; F-circumferential strain; G-radial strain; H-meridional end-systolic stress (ESS); I-circumferential ESS. 
Table 3 Effect of dexrazoxane on changes in echocardiography measures from baseline early after doxorubicin chemotherapy $\left(T_{1}\right)$ according to sex

\begin{tabular}{|c|c|c|c|c|c|}
\hline & Males $(n=48)$ & & Females $(n=37)$ & & \\
\hline Measure & $\begin{array}{l}\text { Effect of } \\
\text { dexrazoxane on } \\
\text { change at } \mathrm{T}_{1}(95 \% \\
\mathrm{Cl})^{*}\end{array}$ & P (dexrazoxane) $\dagger$ & $\begin{array}{l}\text { Effect of dexrazoxane } \\
\text { on change at } \mathrm{T}_{1}(95 \% \\
\mathrm{Cl})^{\star}\end{array}$ & P (dexrazoxane) $\dagger$ & $\mathbf{P}$ (interaction)‡ \\
\hline $\begin{array}{l}\text { Shortening fraction } \\
(\%)\end{array}$ & 0.5 (-2.4 to 3.4$)$ & 0.73 & 5.7 (2.1 to 9.3$)$ & 0.002 & 0.019 \\
\hline $\operatorname{LVIDD}\left(\mathrm{cm} / \mathrm{BSA}^{0.45}\right)$ & $0.1(-0.1$ to 0.3$)$ & 0.49 & $-0.4(-0.7$ to -0.1$)$ & 0.002 & 0.002 \\
\hline LVIDS $\left(\mathrm{cm} / \mathrm{BSA}^{0.45}\right)$ & $-0.03(-0.2$ to 0.1$)$ & 0.73 & $-0.6(-0.8$ to -0.4$)$ & $<0.001$ & $<0.001$ \\
\hline $\begin{array}{l}\text { Posterior wall } \\
\text { thickness }\left(\mathrm{cm} / \mathrm{BSA}^{0.4} \text { ) }\right.\end{array}$ & 0.02 (-0.03 to 0.08$)$ & 0.38 & $0.04(-0.03$ to 0.10$)$ & 0.26 & 0.72 \\
\hline LV mass $\left(\mathrm{g} / \mathrm{BSA}^{1.25}\right)$ & $4.0(-5.2$ to 13.2$)$ & 0.39 & $-7.1(-18.8$ to 4.6$)$ & 0.23 & 0.11 \\
\hline Relative wall thickness & 0.00 (-0.03 to 0.03$)$ & 0.91 & 0.05 (0.01 to 0.08$)$ & 0.015 & 0.046 \\
\hline $\begin{array}{l}\text { Circumferential strain } \\
(\%) \S\end{array}$ & $-3.4(-6.8$ to 0.0$)$ & 0.049 & $-0.6(-5.0$ to 3.8$)$ & 0.78 & 0.27 \\
\hline Radial strain (\%) & 4.6 (-8.2 to 17.5$)$ & 0.47 & 9.9 (-6.9 to 26.8$)$ & 0.24 & 0.58 \\
\hline $\begin{array}{l}\text { Meridional ESS }\left(10^{3}\right. \\
\left.\text { dynes } / \mathrm{cm}^{2}\right)\end{array}$ & -7.9 (-24.0 to 8.2) & 0.33 & $-29.2(-51.1$ to -7.2$)$ & 0.010 & 0.089 \\
\hline $\begin{array}{l}\text { Circumferential ESS } \\
\left(10^{3} \mathrm{dynes} / \mathrm{cm}^{2}\right)\end{array}$ & -7.1 (-23.6 to 9.3$)$ & 0.39 & $-18.9(-41.6$ to 3.9$)$ & 0.10 & 0.36 \\
\hline
\end{tabular}

${ }^{*}$ Difference in the mean change at $T_{1}$ (within 6 months of doxorubicin therapy completion) between subjects who were exposed and not exposed to dexrazoxane, derived from propensity-adjusted multivariable linear regression.

$\dagger \mathrm{P}<0.05$ for the comparison of mean changes by dexrazoxane exposure, performed separately for males and females.

$\ddagger \mathrm{P}$-values for the interaction between sex and dexrazoxane.

§Increases reflect deterioration of circumferential strain.

LVIDS, LV inner dimension in systole

BSA, body surface area; ESS, end-systolic stress; LV, left ventricular; LVIDD, LV inner dimension in diastole.

Locally estimated scatterplot smoothing (LOESS) smoothed curves were derived from unadjusted changes in each measure over time; these curves did not account for within subject correlation. Multivariable linear regression modelled the changes in echocardiography measures separately at $\mathrm{T}_{1}$ and $\mathrm{T}_{2}$, to assess for differences by sex and dexrazoxane exposure status. Sex, dexrazoxane exposure, and the interaction of sex and dexrazoxane exposure were included in each model. These models were adjusted for the propensity to receive dexrazoxane in addition to other prespecified potential confounders: age, race and baseline echocardiographic measure. We created a propensity score for the receipt of dexrazoxane using the subcohort of subjects who were treated before dexrazoxane use was universal. ${ }^{19}$ We used logistic regression to determine a propensity for the receipt of dexrazoxane, and applied this propensity to the overall cohort. The goal here was to adjust for characteristics of the population that influenced receipt of dexrazoxane during the period prior to universal treatment. Predictors in the propensity score model were baseline SF, age, sex, race, body mass index, BSA, sarcoma type, cumulative doxorubicin dose and chest radiation exposure.
Using these models, Wald tests compared mean changes by dexrazoxane exposure according to sex. To assess for effect modification of dexrazoxane's effects by sex, likelihood ratio tests compared full models described above (with the interaction term between sex and dexrazoxane) to reduced models (without the interaction term). Adjustment for multiple comparisons was performed on interaction p-values using a false discovery rate of $5 \%$.

We characterised the temporal relationships between measures at $T_{1}$ with changes at $T_{2}$. Paired t-tests determined whether there were differences in mean values of measures at $T_{1}$ and $T_{2}$. In order to identify early markers of disease, we used multivariable linear regression to determine the associations of changes in each echocardiographic parameter early postdoxorubicin completion (at $T_{1}$ ) with the change in SF 1-2 years postdoxorubicin completion $\left(\right.$ at $\mathrm{T}_{2}$ ). These models were adjusted for baseline SF, baseline echocardiographic parameter of interest, age, race, sex, dexrazoxane exposure, and the interaction between sex and dexrazoxane exposure.

All tests were two-sided, the type I error rate was set at 0.05 , and CIs were at the $95 \%$ level throughout. Statistical 
analyses were performed using STATA V.13.1 (StataCorp) . Graphics were generated using the ggplot2 package in $\mathrm{R}$ V.3.3.3 (R Foundation for Statistical Computing, Vienna, Austria). ${ }^{20}$

\section{RESULTS}

\section{Study population}

Eighty-five subjects were eligible for the study, including 37 females (44\%) and 62 dexrazoxane recipients $(73 \%$; table 1$)$. The average age at baseline was 13.0 years. Overall, $40 \%$ had Ewing sarcoma, 35\% had osteosarcoma, $8 \%$ rhabdomyosarcoma and 17\% non-rhabdomyosarcoma soft tissue sarcoma. On initial presentation, $74 \%$ of tumours were isolated to a single location, $13 \%$ were metastatic to the lungs and $13 \%$ were widely metastatic. The median cumulative dose of doxorubicin therapy was $375 \mathrm{mg} / \mathrm{m}^{2}$ (IQR 375,450 ). Seven subjects received chest radiation, five to primary thoracic sites and two to metastatic lung lesions. Characteristics of subjects who did and did not receive dexrazoxane were similar.

\section{Clinical outcomes}

Nine subjects suffered from cardiac events related to cancer therapy: five developed symptomatic heart failure, three had dose reductions or omissions due to LV dysfunction on echocardiography and one had a significant arrhythmia (medically aborted torsades de pointes). Of these nine subjects, two were male (one treated with and one not treated with dexrazoxane) and seven were female (three treated with and four not treated with dexrazoxane). Four of the five subjects who developed heart failure were treated with afterload reduction medications. Deaths occurred in two of these five subjects with heart failure. One developed a pregnancy while in chronic heart failure with no evidence of cancer; she was lost to follow-up and the circumstances of death were not available (death occurred 4.2 years after diagnosis). Another death was related to care withdrawal in the setting of terminal cancer, sepsis and multiorgan failure (1.6 years after diagnosis). Thirty-two deaths occurred over the duration of available follow-up, at a median of 2.1 years; all other deaths were cancer-related. Overall survival estimated by the Kaplan Meier method at 1, 2 and 5 years was $95 \%$ (95\% CI 88 to 98$), 82 \%$ (95\% CI 72 to 89 ) and $59 \%$ (95\% CI 46 to 69 ), respectively. One secondary malignancy occurred 4.4 years after initial diagnosis in a male subject who received dexrazoxane.

\section{Echocardiographic measures}

In total, 227 echocardiograms were quantitated: 85 at baseline $\left(\mathrm{T}_{0}\right), 85$ after completion of doxorubicin therapy $\left(\mathrm{T}_{1}\right)$ and 571 to 2 years after doxorubicin completion $\left(\mathrm{T}_{2}\right)$. At baseline, echocardiographic parameters were notable for preserved SF and systolic strain indices (table 2). Subjects treated with dexrazoxane had slightly lower SF $(30.1 \%$ vs $33.0 \%, \mathrm{p}=0.025)$, higher LVIDS $(\mathrm{p}=0.002)$, worse circumferential strain $(\mathrm{p}=0.045)$, higher meridional ESS $(\mathrm{p}=0.031)$ and borderline increased circumferential ESS $(\mathrm{p}=0.064)$ in comparison with those not treated with dexrazoxane. Other parameters were similar between groups.

The median time from the baseline echocardiogram to completion of doxorubicin therapy was 6.5 months (IQR 4.9 to 7.1 ), from doxorubicin completion to the $\mathrm{T}_{1}$ echocardiogram was 1.3 months (IQR 0.8 to 2.7 ) and from doxorubicin completion to the $\mathrm{T}_{2}$ echocardiogram was 14.7 months (IQR 12.3 to 17.3). Cancer-related death was the most important cause of reduced data points at $T_{2}$. Baseline clinical characteristics and available echocardiographic variables were similar for those subjects with and without measurements at $T_{2}$ (online supplementary table $1)$.

\section{Changes in echocardiography measures over time and comparison of dexrazoxane effects in males and females}

Unadjusted changes in echocardiographic measures over time are depicted in figures 2 and 3. Propensity-adjusted multivariable linear regression was used to estimate the mean changes in echocardiography measures early after doxorubicin $\left(\right.$ at $\left.\mathrm{T}_{1}\right)$ and 1-2 years postdoxorubicin therapy $\left(\mathrm{T}_{2}\right.$; data not shown $)$. In males not treated with dexrazoxane $(n=15)$, there was an early increase in LVIDS and worsening of circumferential strain. In females not treated with dexrazoxane $(n=8)$, there was evidence of greater dysfunction and worse remodelling, with early and sustained deterioration of SF, circumferential strain and radial strain; increased LVIDD, LVIDS and meridional ESS; and decreased relative wall thickness. These changes were less pronounced with dexrazoxane treatment. In males treated with dexrazoxane $(\mathrm{n}=33)$, slight increases in LVIDD and LVIDS occurred. In females treated with dexrazoxane $(n=29)$, less pronounced deterioration of SF, circumferential strain and radial strain occurred, in addition to a smaller increase in LVIDS.

Using these multivariable regression models, we compared dexrazoxane's effects according to sex at each time point (tables 3 and 4). In males, dexrazoxane was associated with an attenuation of the deterioration of circumferential strain by $3.4 \%$ early after therapy, without significant effects 1-2 years post-therapy. In females, dexrazoxane was associated with an attenuation of the decline in SF (5.7\% difference); the increases in LVIDD, LVIDS and wall stress; and the decreases in relative wall thickness early after therapy, and sustained attenuation of the increases in LVIDD and LVIDS 1-2 years post-therapy. Overall, females and those not treated with dexrazoxane were more likely to develop increased cavity size and decreased cardiac function.

In tests for interactions between sex and dexrazoxane exposure on the changes in parameters of function and remodelling, there was evidence of effect modification on the changes in SF, LVIDD, LVIDS and relative wall thickness early after therapy and on LVIDD and LVIDS 1-2 years post-therapy (all $\mathrm{p}<0.05$ ). After adjustment for multiple comparisons, the interactions remained significant for the relationships between sex and dexrazoxane 
Table 4 Effect of dexrazoxane on changes in echocardiography measures 1-2 years from baseline after doxorubicin chemotherapy $\left(\mathrm{T}_{2}\right)$ according to sex

\begin{tabular}{|c|c|c|c|c|c|}
\hline \multirow[b]{2}{*}{ Measure } & \multicolumn{2}{|l|}{ Males $(n=29)$} & \multicolumn{2}{|l|}{ Females $(n=28)$} & \multirow[b]{2}{*}{ P (interaction)‡ } \\
\hline & $\begin{array}{l}\text { Effect of } \\
\text { dexrazoxane on } \\
\text { change at } \mathrm{T}_{1}(95 \% \\
\mathrm{Cl})^{*}\end{array}$ & P (dexrazoxane)† & $\begin{array}{l}\text { Effect of dexrazoxane } \\
\text { on change at } T_{1}(95 \% \\
C I)^{*}\end{array}$ & P (dexrazoxane)† & \\
\hline $\begin{array}{l}\text { Shortening fraction } \\
(\%)\end{array}$ & $-1.5(-5.3$ to 2.3$)$ & 0.43 & $3.6(-0.7$ to 7.9$)$ & 0.096 & 0.049 \\
\hline $\operatorname{LVIDD}\left(\mathrm{cm} / \mathrm{BSA}^{0.45}\right)$ & $0.1(-0.1$ to 0.4$)$ & 0.42 & $-0.5(-0.7$ to -0.2$)$ & 0.002 & 0.001 \\
\hline LVIDS $\left(\mathrm{cm} / \mathrm{BSA}^{0.45}\right)$ & $0.1(-0.2$ to 0.3$)$ & 0.65 & $-0.5(-0.7$ to -0.3$)$ & $<0.001$ & $<0.001$ \\
\hline $\begin{array}{l}\text { Posterior wall } \\
\text { thickness }\left(\mathrm{cm} / \mathrm{BSA}^{0.4}\right)\end{array}$ & $0.04(-0.02$ to 0.10$)$ & 0.19 & $-0.02(-0.09$ to 0.05$)$ & 0.53 & 0.14 \\
\hline LV mass $\left(\mathrm{g} / \mathrm{BSA}^{1.25}\right)$ & $3.4(-6.2$ to 13.0$)$ & 0.48 & $-8.1(-19.3$ to 3.1$)$ & 0.15 & 0.086 \\
\hline Relative wall thickness & $0.00(-0.04$ to 0.04$)$ & 0.90 & $0.02(-0.03$ to 0.07$)$ & 0.45 & 0.59 \\
\hline $\begin{array}{l}\text { sCircumferential strain } \\
(\%) \S\end{array}$ & $0.0(-3.1$ to 3.0$)$ & 0.98 & $-2.5(-7.1$ to 2.0$)$ & 0.26 & 0.29 \\
\hline Radial strain (\%) & $0.9(-12.7$ to 14.4$)$ & 0.89 & $-4.0(-23.2$ to 15.3$)$ & 0.68 & 0.64 \\
\hline $\begin{array}{l}\text { Meridional ESS }\left(10^{3}\right. \\
\left.\text { dynes } / \mathrm{cm}^{2}\right)\end{array}$ & $14.8(-10.7$ to 40.3$)$ & 0.25 & $-11.9(-41.1$ to 17.3$)$ & 0.41 & 0.094 \\
\hline $\begin{array}{l}\text { Circumferential ESS } \\
\left(10^{3} \text { dynes } / \mathrm{cm}^{2}\right)\end{array}$ & $8.9(-15.1$ to 32.8$)$ & 0.46 & $11.4(-17.2$ to 40.1$)$ & 0.42 & 0.86 \\
\hline
\end{tabular}

*Difference in the mean change at $T_{2}$ (1-2 years after doxorubicin therapy completion) between subjects who were exposed and not exposed to dexrazoxane, derived from propensity-adjusted multivariable linear regression.

$\dagger P<0.05$ for the comparison of mean changes by dexrazoxane exposure, performed separately for males and females.

$\ddagger \mathrm{P}$-values for the interaction between sex and dexrazoxane.

§Increases reflect deterioration of circumferential strain.

BSA, body surface area; ESS, end-systolic stress; LV, left ventricular; LVIDD, LV inner dimension in diastole; LVIDS, LV inner dimension in systole.

on LVIDD ( $\mathrm{p}=0.011)$ and LVIDS $(\mathrm{p}<0.001)$, approached significance for $\mathrm{SF}(\mathrm{p}=0.062)$ early after therapy, and remained significant for LVIDD $(\mathrm{p}=0.006)$ and LVIDS $(\mathrm{p}<0.001) 1-2$ years post-therapy. Overall, these results suggested greater protective effects of dexrazoxane in female children, with similar findings at both time points.

\section{Relationship between early changes and changes $1-2$ years postdoxorubicin}

Finally, we examined the relationship between changes early after doxorubicin $\left(\mathrm{T}_{1}\right)$ and 1-2 years after doxorubicin $\left(\mathrm{T}_{2}\right)$. We first compared values at these two time points (online supplementary table 2). Although there was a nonsignificant initial increase in LV mass was noted early after therapy, there was a small but significant reduction in LV mass by 1-2 years post-therapy in relation to this early change. Other changes in cardiac structure and function were sustained, without further deterioration. We also assessed the associations between early changes in cardiac structure and function with changes in SF 1-2 years after doxorubicin completion to identify potential early markers of global systolic dysfunction (table 5). Here, an early $1 \%$ deterioration of circumferential or radial strain was associated with $\mathrm{SF}$ declines of $0.5 \%$ $(\mathrm{p}=0.010)$ and $0.1 \%(\mathrm{p}=0.025)$ at the late time point, respectively. An early $10 \mathrm{~g} / \mathrm{BSA}^{1.25}$ increase in $\mathrm{LV}$ mass was associated with a decline in SF of $1.2 \% \quad(p=0.021)$ at the late time point. These analyses suggest that the early changes in cardiac structure and function persist, and changes in strain and mass may be early markers of dysfunction.

\section{DISCUSSION}

In this study of children with sarcoma receiving doxorubicin chemotherapy, we report several key findings. First, early after doxorubicin exposure, significant changes in cardiac structure and function occurred in subjects treated with and without dexrazoxane, and these changes persisted 1-2 years post-therapy. Second, greater alterations of cardiac structure and function were found in female children. Third, dexrazoxane mitigated the adverse changes in cardiovascular function and remodelling in both sexes, but protective effects were greater in female children. Fourth, early changes in strain and mass were indicative of late deterioration of SF.

In two recent randomised trials of dexrazoxane cardioprotection with anthracycline therapy in paediatric leukaemia and lymphoma, Lipshultz et al found an improvement in SF with dexrazoxane in females, but 
Table 5 Associations between changes in echocardiography measures early after doxorubicin chemotherapy $\left(T_{1}\right)$ and changes in shortening fraction 1-2 years post chemotherapy $\left(T_{2}\right)$

\begin{tabular}{|c|c|c|}
\hline Echocardiographic measure & $\begin{array}{l}\text { Change in } \\
\text { SF } 1-2 \text { years } \\
\text { post-therapy } \\
\left(\mathrm{T}_{2}\right), \%^{*}\end{array}$ & $\mathbf{P †}$ \\
\hline Shortening fraction (\%) & $0.1(-0.2$ to 0.4$)$ & 0.52 \\
\hline LVIDD (cm/BSA $\left.{ }^{0.45}\right)$ & $-0.8(-5.0$ to 3.4$)$ & 0.71 \\
\hline $\operatorname{LVIDS}\left(\mathrm{cm} / \mathrm{BSA}^{0.45}\right)$ & $-1.0(-6.0$ to 4.1$)$ & 0.70 \\
\hline Posterior wall thickness $\left(\mathrm{mm} / \mathrm{BSA}^{0.4}\right)$ & $-1.6(-3.2$ to 0.0$)$ & 0.054 \\
\hline LV mass $\left(10 \mathrm{~g} / \mathrm{BSA}^{1.25}\right)$ & $\begin{array}{l}-1.1(-2.0 \text { to } \\
-0.1)\end{array}$ & 0.025 \\
\hline Relative wall thickness (0.01 units) & $-0.2(-0.5$ to 0.1$)$ & 0.22 \\
\hline Circumferential strain $(\%) \dagger$ & $\begin{array}{l}-0.5(-0.8 \text { to } \\
-0.1)\end{array}$ & 0.010 \\
\hline Radial strain (\%) & 0.1 (0.0 to 0.2$)$ & 0.025 \\
\hline Meridional ESS $\left(10^{*} 10^{3}\right.$ dynes $\left./ \mathrm{cm}^{2}\right)$ & $-0.1(-0.8$ to 0.5$)$ & 0.68 \\
\hline Circumferential ESS $\left(10^{*} 10^{3}\right.$ dynes $\left./ \mathrm{cm}^{2}\right)$ & $-0.1(-0.7$ to 0.5$)$ & 0.85 \\
\hline
\end{tabular}

${ }^{*}$ Percent change in $\mathrm{SF}$ at $\mathrm{T}_{2}$ for each unit increase in echocardiography measure at $T_{1}$ in multivariable linear regression. $\dagger P$ value for the association of the change in SF at $T_{2}$ for each unit increase in echocardiography measure at $T_{1}$ in multivariable linear regression.

łlncreases reflect a deterioration of circumferential strain.

BSA, body surface area; ESS, end-systolic stress; LV, left ventricular; LVIDD, LV inner dimension in diastole; LVIDS, LV inner dimension in systole.

not males with a significant interaction, and Asselin et al found significant improvement in SF with dexrazoxane overall. ${ }^{9}{ }^{10}$ Our results are additive to these and other paediatric cardio-oncology studies in several ways. First, we focused on a sarcoma population, a disease group understudied in this context, and a group where dexrazoxane's cardioprotective effects are of particular interest given the high anthracycline dosage. Second, we found that changes in cardiac structure and function occurred early after high-dose anthracyclines and persisted to 1-2 years postdoxorubicin. These findings are also observed in the setting of dexrazoxane, although adverse changes are attenuated. Third, we studied an expanded set of measures, including strain, and found that circumferential strain may provide additional information to understand the functional perturbations that occur in children exposed to anthracyclines.

In our study, female children experienced greater cardiac abnormalities than male children, and dexrazoxane's effects were greater in females. The increased vulnerability of female children to anthracyclines has been previously reported and attributed to differences in pharmacokinetics, possibly related to differences in body fat composition, though other factors may also play a role. ${ }^{1112} 2122$ The mechanisms by which dexrazoxane may provide greater cardioprotection to females have not been established and it is possible that dexrazoxane may mitigate cardiotoxicity similarly in both sexes, but females derive more benefit due to greater underlying susceptibility. Our findings suggest that with further research, patient sex may be considered in the clinical decision-making process of whether to use dexrazoxane.

Finally, our data provide insight into early cardiac remodelling after anthracyclines in children. Similar to prior studies, our subjects had reductions in SF and strain, and early increases in cavity diameter. ${ }^{10}{ }^{23}$ In addition to identifying these changes, we found that early increases in LV mass and deterioration of circumferential and radial strain were indicative of deterioration in SF at 1-2 years post-therapy. Prognostic associations of early changes in strain indices have been found in a number of studies in adults. ${ }^{162}$ Our data, in conjunction with these adult studies, provide support for the use of circumferential strain as an adjunct measure in the early monitoring of paediatric patients receiving anthracycline therapy. The finding that early mass increases may be a pathologic finding after anthracycline therapy is consistent with recent studies of adults exposed to anthracyclines, ${ }^{16} 25$ but differs from prior studies in long-term cancer survivors, which demonstrate reductions in LV mass. ${ }^{5}$ It is possible that myocardial oedema plays a role in these early changes, ${ }^{26}$ and it is notable that there was a subsequent decrease in mass by the later time point, which may reflect an evolving process.

We acknowledge the potential limitations of our study. A lack of dedicated prospective imaging precluded the ability to assess longitudinal strain or LV ejection fraction, although SF is the most widely used measure in paediatric cardiology. Images were analysed at the archived frame rate of 30 frames per second, which could result in some limitations in strain analyses specifically, although prior studies have demonstrated that this frame rate is adequate for analyses. ${ }^{13}{ }^{14}$ Despite our careful adjustments for measured confounders, bias due to unmeasured factors is possible. In addition, although we adjusted for multiple comparisons in our primary interaction analyses, other statistical tests were susceptible to type I error related to multiple comparisons. Sample size and length of follow-up constraints precluded the ability to assess long-term cardiac outcomes with dexrazoxane, including the development of heart failure, oncologic outcomes or mortality. Finally, this study was conducted in a single tertiary care centre, and our results may not be generalisable.

In conclusion, high-dose anthracycline therapy continues to pose a risk of cardiotoxic effects in the postdexrazoxane era. Female children are more susceptible to these effects and may derive greater cardioprotective benefit from dexrazoxane. These results have potential implications for the care of children receiving anthracyclines. 
Author affiliations

${ }^{1}$ Department of Pediatrics, University of California San Diego School of Medicine, San Diego, California, USA

${ }^{2}$ Rady Children's Hospital San Diego, San Diego, California, USA

${ }^{3}$ Biostatistics, Epidemiology and Informatics, University of Pennsylvania, Philadelphia, Pennsylvania, USA

${ }^{4}$ Department of Medicine, University of Pennsylvania, Philadelphia, Pennsylvania, USA

${ }^{5}$ Department of Pediatrics, Children's Hospital of Philadelphia, Philadelphia, Pennsylvania, USA

${ }^{6}$ Department of Medicine, University of Pennsylvania, Philadelphia, Pennsylvania, USA

${ }^{7}$ Division of Cardiology, The Children's Hospital of Philadelphia, Philadelphia, Pennsylvania, USA

${ }^{8}$ Department of Population Sciences, City of Hope National Medical Center, Duarte, California, USA

${ }^{9}$ Department of Pediatrics, University of Chicago, Chicago, Illinois, USA

${ }^{10}$ Abramson Cancer Center, Philadelphia, Pennsylvania, USA

${ }^{11}$ Department of Cardiology, University of Pennsylvania, Philadelphia, Pennsylvania, USA

Contributors HKN: conceptualisation, methodology, data curation, formal analysis, writing-original draft. MEP: methodology, formal analysis, writing-review/ editing. NK, AP, RBW and AVD: conceptualisation, data curation, writing-review/ editing. SB and TP: methodology, data curation, writing-review/editing. LM-R and SHA: Methodology, writing-review/editing. BK: conceptualisation, data curation, methodology, formal analysis, funding acquisition, writing-review/editing.

Funding This work was supported by the National Institutes of Health (T32HD060550 [Narayan], R01-HL118018 [Ky]).

Competing interests None declared.

Patient consent for publication Not required.

Provenance and peer review Not commissioned; externally peer reviewed.

Data sharing statement Data are available upon reasonable request.

Open access This is an open access article distributed in accordance with the Creative Commons Attribution Non Commercial (CC BY-NC 4.0) license, which permits others to distribute, remix, adapt, build upon this work non-commercially, and license their derivative works on different terms, provided the original work is properly cited, appropriate credit is given, any changes made indicated, and the use is non-commercial. See: http://creativecommons.org/licenses/by-nc/4.0/.

\section{REFERENCES}

1. Krischer JP, Epstein S, Cuthbertson DD, et al. Clinical cardiotoxicity following anthracycline treatment for childhood cancer: the pediatric Oncology Group experience. J Clin Oncol 1997;15:1544-52.

2. Armstrong GT, Oeffinger KC, Chen Y, et al. Modifiable risk factors and major cardiac events among adult survivors of childhood cancer. $J$ Clin Oncol 2013;31:3673-80.

3. Janeway KA, Grier HE. Sequelae of osteosarcoma medical therapy: a review of rare acute toxicities and late effects. Lancet Oncol 2010;11:670-8.

4. Brown TR, Vijarnsorn C, Potts J, et al. Anthracycline induced cardiac toxicity in pediatric Ewing sarcoma: a longitudinal study. Pediatr Blood Cancer 2013;60:842-8.

5. Lipshultz SE, Lipsitz SR, Sallan SE, et al. Chronic progressive cardiac dysfunction years after doxorubicin therapy for childhood acute lymphoblastic leukemia. JCO 2005;23:2629-36.

6. Armstrong GT, Joshi VM, Ness KK, et al. Comprehensive Echocardiographic Detection of Treatment-Related Cardiac Dysfunction in Adult Survivors of Childhood Cancer. J Am Coll Cardiol 2015:65:2511-22.

7. Rajagopalan S, Politi PM, Sinha BK, et al. Adriamycin-induced free radical formation in the perfused rat heart: implications for cardiotoxicity. Cancer Res 1988;48:4766-9.
8. Lyu YL, Kerrigan JE, Lin C-P, et al. Topoisomerase Ilbeta mediated DNA double-strand breaks: implications in doxorubicin cardiotoxicity and prevention by dexrazoxane. Cancer Res 2007;67:8839-46.

9. Lipshultz SE, Scully RE, Lipsitz SR, et al. Assessment of dexrazoxane as a cardioprotectant in doxorubicin-treated children with high-risk acute lymphoblastic leukaemia: long-term followup of a prospective, randomised, multicentre trial. Lancet Oncol 2010;11:950-61.

10. Asselin BL, Devidas M, Chen L, et al. Cardioprotection and safety of dexrazoxane in patients treated for newly diagnosed T-cell acute lymphoblastic leukemia or advanced-stage lymphoblastic nonHodgkin Iymphoma: a report of the children's Oncology Group randomized trial pediatric Oncology Group 9404. J Clin Oncol 2016;34:854-62

11. Silber JH, Jakacki RI, Larsen RL, et al. Increased risk of cardiac dysfunction after anthracyclines in girls. Med Pediatr Oncol 1993;21:477-9.

12. Lipshultz SE, Lipsitz SR, Mone SM, et al. Female sex and higher drug dose as risk factors for late cardiotoxic effects of doxorubicin therapy for childhood cancer. N Engl J Med 1995;332:1738-44.

13. Zhang KW, French B, May Khan A, et al. Strain improves risk prediction beyond ejection fraction in chronic systolic heart failure. $J$ Am Heart Assoc 2014;3:e000550.

14. Hung C-L, Verma A, Uno H, et al. Longitudinal and circumferential strain rate, left ventricular remodeling, and prognosis after myocardial infarction. J Am Coll Cardiol 2010;56:1812-22.

15. Lopez L, Colan SD, Frommelt PC, et al. Recommendations for quantification methods during the performance of a pediatric echocardiogram: a report from the pediatric measurements writing group of the American Society of echocardiography pediatric and congenital heart disease Council. J Am Soc Echocardiogr 2010;23:465-95.

16. Narayan HK, Finkelman B, French B, et al. Detailed echocardiographic phenotyping in breast cancer patients: associations with ejection fraction decline, recovery, and heart failure symptoms over 3 years of follow-up. Circulation 2017;135:1397-412.

17. Reichek N, Wilson J, St John Sutton M, et al. Noninvasive determination of left ventricular end-systolic stress: validation of the method and initial application. Circulation 1982;65:99-108.

18. Lopez L, Colan S, Stylianou M, et al. Relationship of Echocardiographic $Z$ Scores Adjusted for Body Surface Area to Age, Sex, Race, and Ethnicity: The Pediatric Heart Network Normal Echocardiogram Database. Circ Cardiovasc Imaging 2017;10.

19. Rosenbaum PR. Design of observational studies. 1st edn. New York: Springer, 2012.

20. Wickham H. Ggplot2: elegant graphics for data analysis. New York: Springer, 2009.

21. Thompson PA, Rosner GL, Matthay KK, et al. Impact of body composition on pharmacokinetics of doxorubicin in children: a Glaser pediatric research network study. Cancer Chemother Pharmacol 2009;64:243-51.

22. Dobbs NA, Twelves CJ, Gillies H, et al. Gender affects doxorubicin pharmacokinetics in patients with normal liver biochemistry. Cancer Chemother Pharmacol 1995;36:473-6.

23. Mavinkurve-Groothuis AMC, Marcus KA, Pourier M, et al. Myocardial 2D strain echocardiography and cardiac biomarkers in children during and shortly after anthracycline therapy for acute lymphoblastic leukaemia (ALL): a prospective study. Eur Heart J Cardiovasc Imaging 2013;14:562-9.

24. Thavendiranathan P, Poulin F, Lim K-D, et al. Use of myocardial strain imaging by echocardiography for the early detection of cardiotoxicity in patients during and after cancer chemotherapy: a systematic review. J Am Coll Cardiol 2014;63:2751-68.

25. Tan TC, Bouras S, Sawaya H, et al. Time Trends of Left Ventricular Ejection Fraction and Myocardial Deformation Indices in a Cohort of Women with Breast Cancer Treated with Anthracyclines, Taxanes, and Trastuzumab. J Am Soc Echocardiogr 2015;28:509-14

26. Farhad H, Staziaki PV, Addison D, et al. Characterization of the changes in cardiac structure and function in mice treated with anthracyclines using serial cardiac magnetic resonance imaging. Circ Cardiovasc Imaging 2016;9:e003584. 